DOI:10.12731/2070-7568-2020-4-328-346

УДК 338.001.36

\title{
КВАЛИМЕТРИЧЕСКИЙ ПОДХОД К РЕЙТИНГОВАНИЮ СТАРТАПОВ
}

\section{Олейник А.Г., Ахмедова М.Р.}

Данная статья посвящена исследованию и применению квалиметрического подхода при анализе и рейтинговании стартапов. Существует множество методик оченки стартапов, однако квалиметрический подход имеет преимущества перед ними, т.к. он составляет критерии ранжсрованных списков, которые определяют интегральное качество для каждого стартапа с изелью составления рейтинга. Авторами были выявлены все базовые показатели стартапов, а также при помощи методов квалиметрии данные показатели были сведены вместе, преобразованы для того, чтобы обеспечить достоверность и точность каждого из результатов данных показателей. На основании проведенного исследования были вылвлены основные этапь работ квалиметрического анализа, которье необходимо провести при рейтинговании стартапов и выделены значимости и весомости исследования по каждому базисному показателю.

Цель - применение квалиметрического подхода при рейтинговании стартапов.

Метод и методология проведения работы: комплекс методов теоретического обоснования (описание, сравнение), квалиметрический метод, экспертный метод.

Результаты: выявлены основные базисные показатели стартапов, построено дерево свойств рейтингования стартапов и поддеревья каждого из базисных показателей, назначены интерваль изменений каждого базисного показателя и рассчитаны их показатели весомости при помощии экспертного метода.

Область применения результатов: полученные в рамках настоящего исследования результаты иелесообразно использовать 
при анализе различных видов стартапов с ичелью рейтингования $и$ выявления их эффективности.

Ключевые слова: квалиметрический анализ; рейтингование стартапов; анализ стартапов; эффективность стартапов; качественный анализ показателей стартапа.

\section{QUALIMETRIC APPROACH TO RATING STARTUPS}

\section{Oleynik A.G., Akhmedova M.R.}

This article is devoted to the research and application of the qualimetric approach in the analysis and rating of startups. There are many methods of evaluating startups, but the qualimetric approach has advantages over them, because it creates the criteria for ranked lists that determine the integral quality for each startup in order to compile a rating. The authors identified all the basic indicators of startups, and using qualimetry methods, these indicators was brought together and transforming in order to ensure the reliability and accuracy of every results of these indicators. Based on the conducted research, the main stages of qualimetry work that need to was be carried out in rating startups were identified and the signifcance and weight of the research for each basic indicator were highlighted.

Purpose applying the qualimetric approaches for rating startups

Methodology complex of theoretical justification methods (description, comparison), qualimetry methods, expert method

Results: the basic indicators of startups was identified, a tree of startup rating properties and sub trees of each of the basic indicators are constructed, intervals of changes for each basic indicator are assigned, and their weight indicators are calculated using the expert method.

Practical implications: the results of the research can be using in the analysis of various types of startups in order to rank and identify their effectiveness.

Keywords: qualitative analysis; rating of startups; analysis; startups; effectiveness of startups; qualitative analysis of the indicators of a startup. 


\section{Введение}

В настоящее время существует сложность в оценке качества новых проектов (стартапов), т.к. инновации всегда имеют большую степень неопределенности и изменчивости. Поэтому для эффективной оценки стартапов лучше использовать многокритериальные методики, которые оценивают качество стартапов поэтапно и для этого на каждом этапе вводится самостоятельный критерий, т.е. описываются все показатели стартапа [9, с. 55] [16, с. 114].

Одна из таких многокритериальных методик - квалиметрия, которая была разработана еще в 1968 году Г.Г. Азгальдовым [4, с. 10] и является одним из самых надежных методов оценивания качества.

Поскольку, методы применяемые в квалиметрии помогают оценивать качество не только продукции, но и сложных процессов, то на данный момент квалиметрический анализ можно с наиболее вероятным успехом применить к оценке и отбору стартапов.

\section{Цель исследования}

Целью исследования является разработка методики оценки стартапов на основе квалиметрического подхода.

\section{Материалы и методы исследования}

Материалы данного исследования основаны на понятии квалиметрического моделирования, введенного Азгальдовым в 1968 году, также исследование основано на материалах электронных библиотек и зарубежных статей по квалиметрическому анализу инновационных проектов и стартапов. В процессе исследования был использован комплекс методов теоретического обоснования (описание, сравнение), квалиметрический метод, экспертный метод.

\section{Результаты исследования}

Вообще стартап - это временная структура, которая направлена на поиск и реализацию масштабной бизнес-идеи, при этом от грамотной оценки стартапов на начальной стадии развития, зависит успех реализации идеи в дальнейшей перспективе. Однако на дан- 
ный момент существует множество методик, оценивающих стартапы, например: методика скоринга, модель Ave Maria, стоимостные методы оценки [5, с. 479]. Поэтому, что увидеть преимущества квалиметрического метода над остальными построим таблицу сравнения методологий оценки стартапов [3, с. 7], таблица 1.

Таблица 1.

\section{Сравнение методов оценки стартапов}

\begin{tabular}{|l|l|}
\hline \multicolumn{1}{|c|}{$\begin{array}{c}\text { Методы анализа } \\
\text { стартапов }\end{array}$} & \multicolumn{1}{|c|}{ Сравнение с квалиметрическим анализом } \\
\hline Метод скоринга & $\begin{array}{l}\text { Отличие модели скоринга и квалиметрического } \\
\text { подхода, заключается в том, что существенным } \\
\text { недостатком первого метода является произвольный } \\
\text { выбор показателей сравнения стартапов и } \\
\text { их значимости, а также неопределенность } \\
\text { объекта исследования, где в отличие от этого } \\
\text { квалиметрический анализ логически обьясняет } \\
\text { каждый свой выбор критериев. [17, с. 2983] }\end{array}$ \\
\hline $\begin{array}{l}\text { Стоимостной метод } \\
\text { оценки }\end{array}$ & $\begin{array}{l}\text { Отличие метода оценивания стоимости и квалиметрии, } \\
\text { состоит в том, что использование квалиметрического } \\
\text { анализа снижает совокупные издержки по } \\
\text { рейтингованию стартапов и снижает неопределенность } \\
\text { показателей за счет научного подхода к критерием } \\
\text { оценки показателей и составлению дерева свойств [19] }\end{array}$ \\
\hline Модель Avе Магіа & $\begin{array}{l}\text { Модель Ауе Магіа также, как и квалиметрическая } \\
\text { методология полезна при оценке нескольких стартапов, } \\
\text { однако она, в отличие от квалиметрического подхода, } \\
\text { не дает численных оценок по каждому базовому } \\
\text { показателю. [19] }\end{array}$ \\
\hline
\end{tabular}

Исходя из таблицы 1 можно сделать вывод, что приоритет квалиметрического подхода перед остальным для оценивания стартапов заключается главным образом в критериях ранжированных списков, которые определяют интегральное качество для каждого стартапа с целью составления рейтинга.

Также квалиметрический подход при ранжировании стартапов, может быть эффективно реализован в большей степени на стадии развития проекта, т.к. данный анализ помогает снизить неопределённость инновационности стартапа, за счет построения иерархической структурной схемы (дерева свойств) и выделения каждого показателя как совокупности части данной системы. 
При этом квалиметрические методики помогают ранжировать стартапы по интегральному качеству, где каждый показатель будет соответствовать нормам обоснованности и сравнимости. Благодаря квалиметрическому анализу стартапов, не только можно эффективно составить рейтинг, но и минимизировать издержки при проведении итогов за счет всестороннего оценивания, которое включает не только количественные экономические показатели, но и качественные не экономические процессы, поэтому квалиметрический анализ включает в себя и критерии оценки качества каждого рассматриваемого показателя и критерии эффективности стартапов.

Также стратегические цели квалиметрического анализа, могут включать не только измерение как количественных, так и качественных показателей стартапов, но также и сравнение результативности каждого из этих показателей по интегральному качеству.

Рассмотрим квалиметрический метод рейтингования стартапов. Для начала необходимо сказать, что каждый стартап имеет свои затраты, стадии развития, сферы применения, свой собственный продукт. Однако квалиметрический анализ позволяет учитывать все показатели, которые входят в стартап. Также при помощи методов квалиметрии данные показатели будут сведены вместе, преобразованы для того, чтобы обеспечить достоверность и точность каждого из результатов данных показателей.

Однако, как и любая методология квалиметрический анализ имеет ряд работ которые необходимо выполнять в следующем порядке [16, с. 114]:

1. Выбор базовых показателей;

2. Составление иерархической структурной схемы (дерево свойств);

3. Определение способа нахождения весомостей (стоимостной, эвристический, экспертный, вероятностный, комбинированный);

4. Назначение интервала изменения значений Рі каждого показателя;

5. Назначение веса показателя качества Рі.Исходя из выше перечисленных работ, которые необходимо выполнить при 
проведении квалиметрического анализа, для оценки и ранжирования стартапов первоначально необходимо выделить базовые показатели, которые комплексно смогли бы охарактеризовать степень эффективности стартапа. Для этого были выбраны такие показатели, как продукт, рынок, финансирование - эти показатели характерны для анализа любых видов проектов.

Базовый показатель - продукт для стартапа, будет рассматриваться со стороны инновационности и степени конкурентоспособности, т.к. качественный анализ этих показателей поможет оценить степень эффективности создания продукта для потребителя [7, с. 133].

Базовый показатель - рынок, как и для любого проекта, должен характеризовать качественный анализ рынка стартапа, однако для стартапов важно учитывать не только сам рынок, но и маркетинговый план при внедрении продукта.

Базовый показатель - финансирование, также характерен для любого проекта, качественный анализ размера и эффективности инвестиции поможет грамотно проранжировать стартапы, но следует также оценить возможность надежности инвестиций, т.к. каждый стартап имеет свою степень рисков на каждом этапе реализации.

Также необходимо выделить базовые показатели, такие как команда и риски, более характерные именно для инновационных проектов.

Базовый показатель - команда, необходим при анализе стартапа, т.к. оценка взаимодействия членов команды, может охарактеризовать качество реализации стартапа еще на начальном уровне его разработки и реализации.

Базовые показатель - риски, не менее важно учитывать при ранжировании стартапов. Высокорисковые проекты носят обычно инновационный характер и степень оценивания рисков очень важна для дальнейшей их реализации, т.к. любой неучтенный риск тем более на стадии реализации проекта, может привести к невозможности выхода стартапа на рынок [8, с. 13].

После выбора базовых показателей, в квалиметрическом анализе необходимо составить иерархическую структуру или дерево 
свойств. Для наглядного представления построим дерево свойств базовых показателей, рисунок 1 .

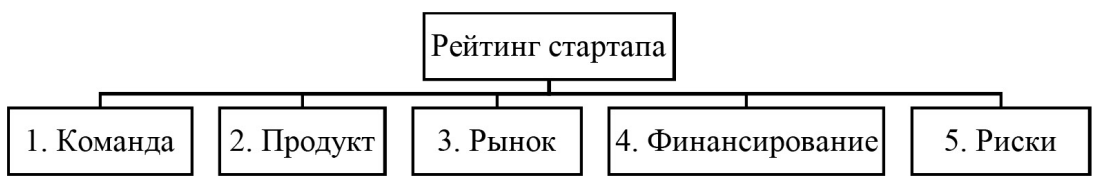

Рис. 1. Дерево общих свойств стартапа

После выделения и построения дерева общих свойств, необходимо выделить специфику каждого базисного показателя и представить в виде иерархической структуры (поддерево свойств назначения), это необходимо для того чтобы квалиметрический анализ включал показатели четко рейтингующие каждый стартап, для более точного и надежного анализа. Так поддерево свойств назначения показателей команды представлено на рисунке 2.

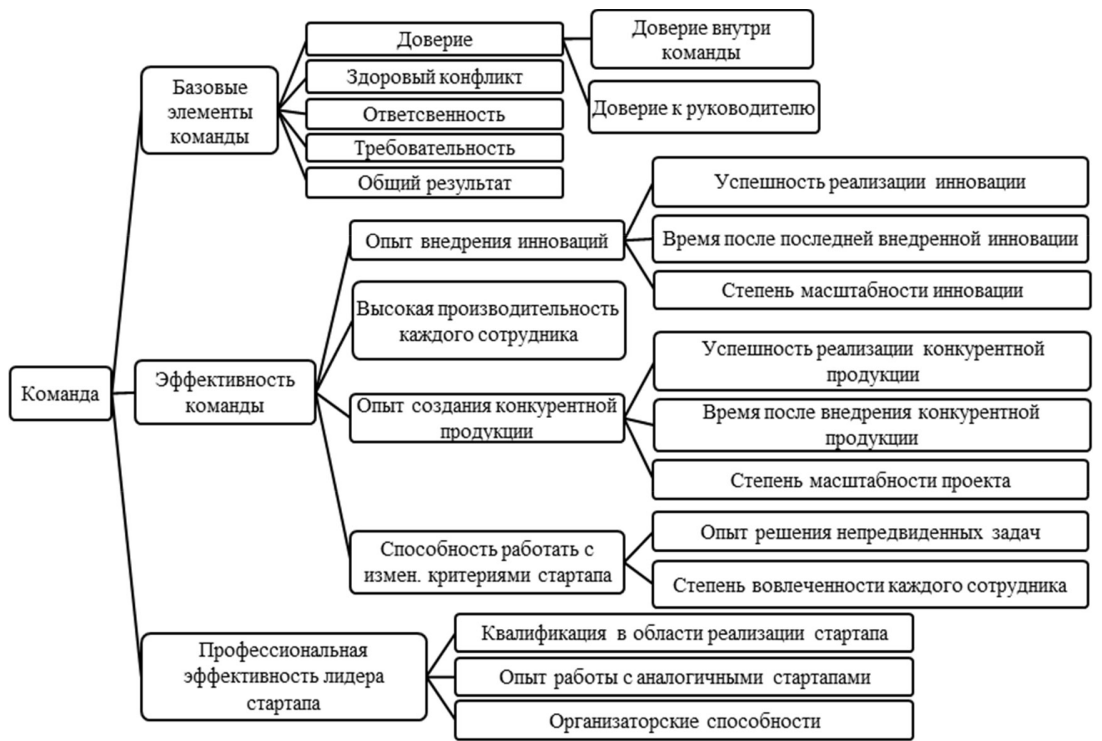

Рис. 2. Поддерево свойств назначения показателей команды

На рисунке 2 представлены показатели характеризующие уровень команды стартапа, для этого были выделены основные критерии, такие как: 
1. Базовые элементы команды - данные показатели характеризуют (доверие в команде его, ответственность и т.д.), где благодаря этим показателя можно качественно оценить степень надежность команды [6, с. 688];

2. Эффективность команды - в данном показатели учитывается опыт команды в реализации похожих проектов, а также их умение находить выход из постоянно изменяющихся критериев стартапов;

3. Профессиональная эффективность лидера стартапа - данный показатель также необходимо учитывать при рейтинговании стартапов, т.к. при помощи него можно оценить квалификацию и опыт работ лидера, как самого главного звена команды проекта.

После составления поддерева свойств назначения команды стартапа, необходимо представить показатели продукта для анализа и рейтингования стартапов, данные показатели представлены на рисунке 3 .

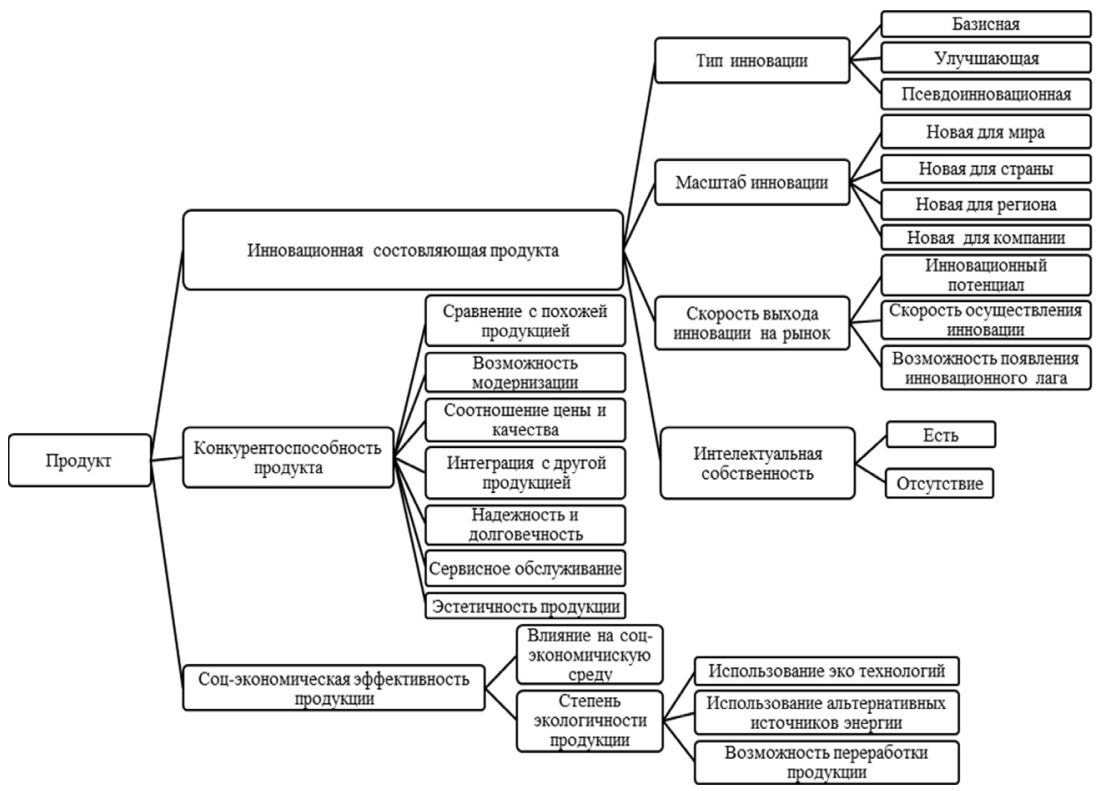

Рис. 3. Поддерево свойств назначения показателей продукта

На рисунке 3 представлены показатели характеризующие продукцию стартапа, где для этого были выделены критерии: 


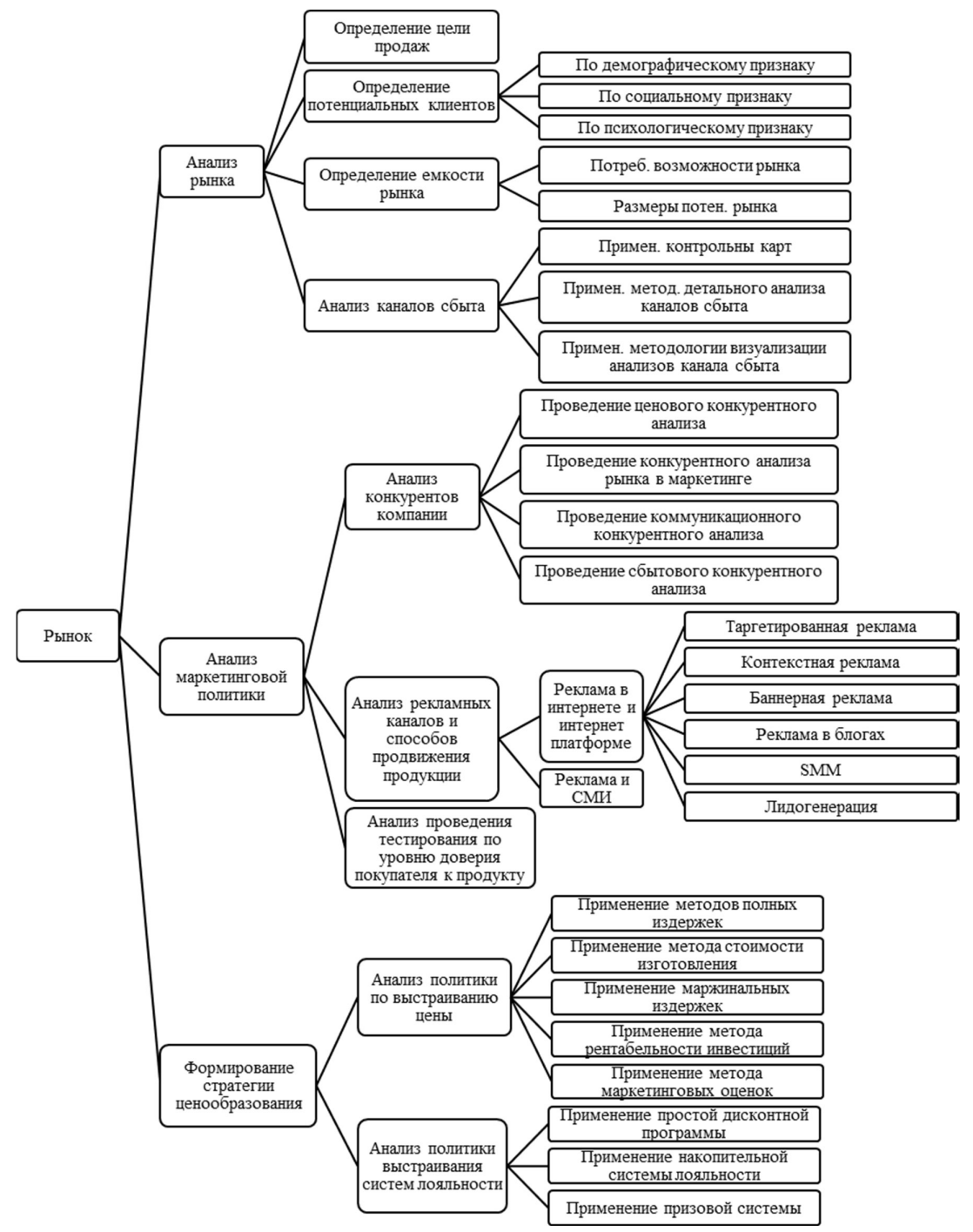

Рис. 4. Поддерево свойств назначения показателей рынка

1. Инновационная составляющая продукта - данный показатель важен при оценке стартапов, т.к. степень инновационности один из главных составляющих успешности нового проекта [12]; 
2. Конкурентоспособность продукта - данный показатель характерен для любого вида проекта, т.к. при помощи него можно сказать о преимуществах продукции стартапа перед другими [15, с. 625];

3. Социально-экономическая эффективность продукции - данный показатель был выделен, т.к. стартап, как и любо современный проект в рамках последних тенденций рынка на экологичность и социальность должен удовлетворять данным критериям и превосходить другие проекты.

Далее необходимо рассмотреть базовый показатель - рынок и выделить критерии для анализа данного показателя, представив из в виде поддерева свойств назначения рынка, рисунок 4.

На рисунке 4 представлены показатели характеризующие рынок стартапа, где для этого были выделены:

1. Анализ рынка - данный показатель характеризует емкость рынка, цель продаж, потенциального клиента, анализирует каналы сбыта, т.е. при помощи данного показатели мы можем понять подойдет ли продукт стартапа рынку или нет и сравнить степень успешности данного стартапа на рынке по сравнению с другими проектами [13, с. 3];

2. Анализ маркетинговой стратегии - данный показатель важен при рейтинговании стартапов, т.к. новый продукт всегда нужно грамотно и вовремя продвинуть на рынок, поэтому анализ рекламных каналов сбыта, конкурентоспособности продукции, а также проведение тестирования продукта среди потенциальных клиентов рынка, является важным показателем при выборе эффективного стартапа;

3. Формирование стратегии ценообразования - данный показать необходимо также включить в критерии анализа стартапов, т.к. грамотная политика в области выстраивания цены, также важна для новой продукции на рынке, т.к. без нее возрастает риск неэффективности продажи продукции стартапа.

Далее необходимо рассмотреть базовый показатель - финансирование и выделить критерии для анализа данного показателя, представив из в виде поддерева свойств назначения рынка, рисунок 5 .

На рисунке 5 представлены показатели характеризующие финансирование стартапа, где для этого были выделены критерии: 


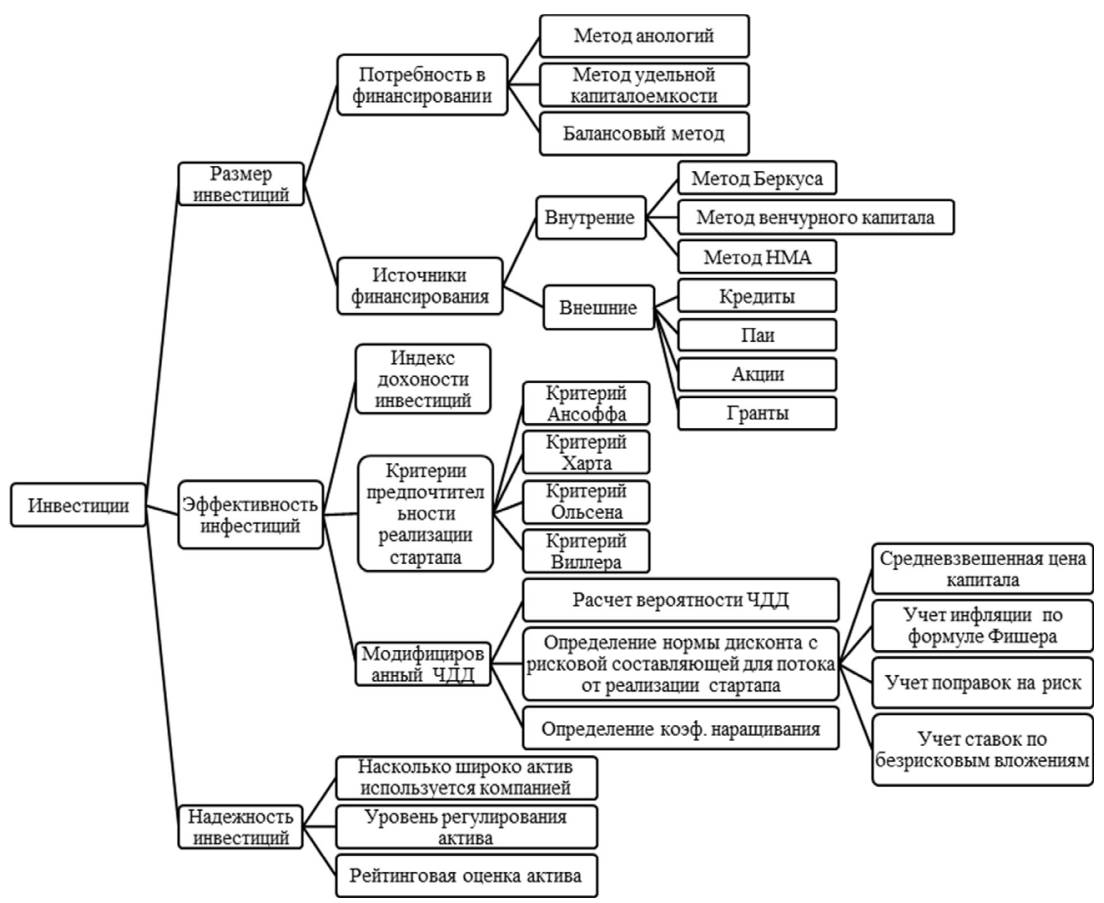

Рис. 5. Поддерево свойств назначения показателей финансирования

1. Размер инвестиций - данный показатель необходим для анализа каждого проекта, поэтому анализ стартапов по данному критерию также необходимо производить, чтобы понять правильно ли рассчитана потребность в финансировании для проекта [14, с.64];

2. Эффективность инвестиций - данный показатель также важен при рейтинговании стартапов, т.к. именно он показывает индекс доходности от капитальных вложений в стартап [10, с.161];

3. Надежность - данный показатель важен при анализе стартапов, т.к. стартап подразумевает под собой реализацию совершенно нового продукта, поэтому анализ активов стартапов один из главных критериев его рейтингования.

Далее необходимо рассмотреть базовый показатель - риски и выделить критерии для анализа данного показателя, представив из в виде поддерева свойств назначения рисков, рисунок 6 . 


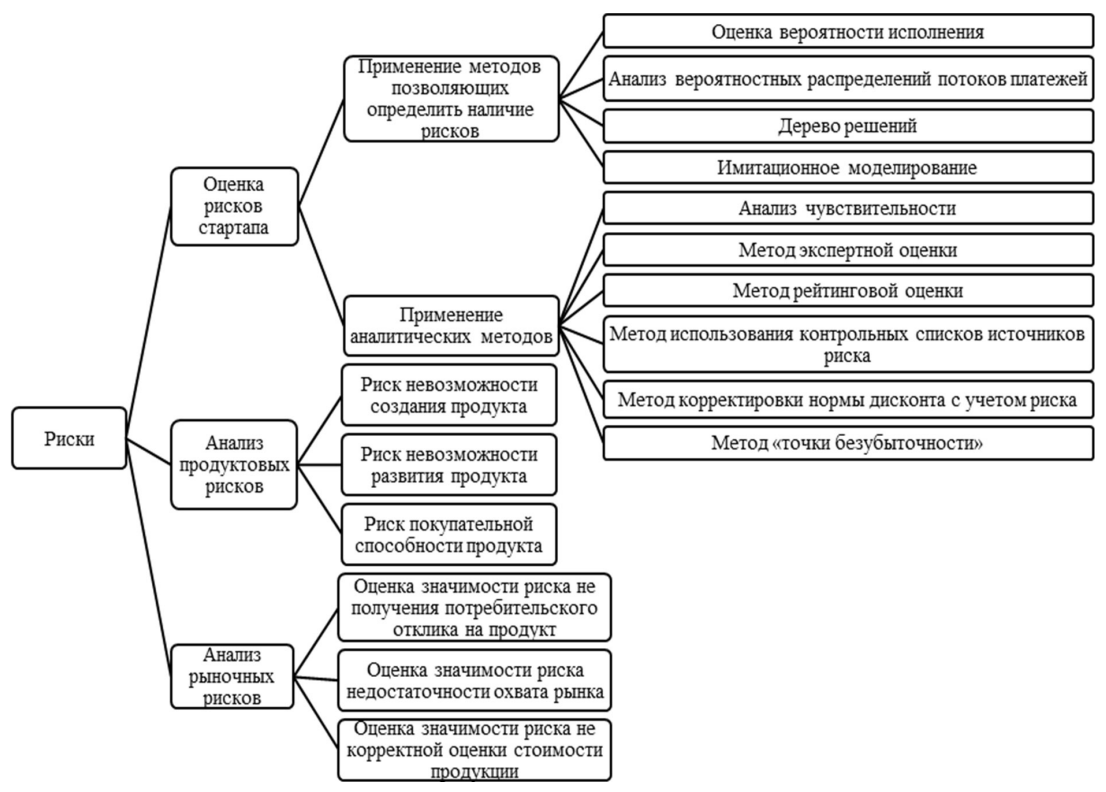

Рис. 6. Поддерево свойств назначения показателей рисков

На рисунке 6 представлены показатели характеризующие риски стартапа, где для этого были выделены критерии:

1. Оценка рисков стартапов - данный критерий помогает понять насколько грамотно оценены риски стартапов, т.к. из-за своей новизны каждый стартап имеет повышенные риски при его реализации $[3$, с. 7];

2. Анализ продуктовых рисков - критерий также важен при рейтинговании стартапов, т.к. необходимо учитывать, что риск не создания продукта очень высок и от оценки данного критерия напрямую зависит степень оценивания стартапа;

3. Анализ рыночных рисков - данный критерий также важен, как и анализ продуктов рисков, т.к. продукция проекта для рынка совершенно новая и необходимо грамотно рассчитать риски по потребительскому отклику на продут стартапа.

Далее после выбора базовых показателей при анализе стартапа и после построения иерархической схемы (дерева базовых показа- 
телей и поддеревьев каждого из них), необходимо при проведении квалиметрического анализа определить способ нахождения коэффициента весомостей, для этого воспользуемся экспертным методом, т.к. оценивание и рейтингование стартапов процесс достаточно сложный и должен опираться на весомое мнение эксперта, поэтому для того чтобы рассчитать Рі значений каждого базисного критерия воспользуемся методом экспертного определения коэффициентов весомости. Так коэффициент весомости $i$-го базисного показателя стартапа будет определяться по формуле:

$$
P \mathrm{i}=\frac{\mathrm{Ai}}{\sum A \mathrm{i}}
$$

где $\mathrm{Pi}$ - коэффициент весомости $i$-го базисного показателя стартапа [11];

$\mathrm{Ai}$ - измеренное значение $i$-ой важности показателей стартапа;

$\sum \mathrm{Ai}$ - суммарное значение $i$-ых важностей показателей стартапа.

После определения способа нахождения коэффициента весомостей необходимо назначить интервалы изменения значений Pi каждого показателя, для этого необходимо обратится к измеренным оценкам важности показателей стартапов (Ai), с этой целью обратимся к экспертному мнению автора метода скоринга - Биллу Пейну, который оценивает каждый показатель так [18, с. 15] [19]:

1. Наличие сильной управляющей команды: 0-30\%;

2. Размер рынка: 0-25\%;

3. Новизна продукта и технологии: $0-15 \%$

4. Конкурентные окружение: $0-10 \%$;

5. Маркетинг, каналы продаж, партнерство: 0-10\%

6. Потребность в дополнительных инвестициях: 0-5\%

7. Другие факторы: $0-5 \%$

Исходя из оценки показателей стартапов Биллом Пейном, рассчитаем Аі для каждого базисного показателя выделенного в данной работе. Для этого возьмем крайнее значение показателей в экспертной оценке Билла Пейна и рассчитаем его в качестве оценки по 5-бальной шкале, таблица 2: 
Таблицуа 2.

Расчет Аі показателей

\begin{tabular}{|l|l|l|}
\hline $\begin{array}{c}\text { Базисный } \\
\text { показатель } \\
\text { стартапа }\end{array}$ & \multicolumn{1}{|c|}{$\begin{array}{c}\text { Аі по 5-бальной } \\
\text { шкале }\end{array}$} & Критерии оценки по методу Билла Пейна \\
\hline Команда & $5 * 0,3=1,5$ балла & 1. Наличие сильной управляющей команды: \\
\hline Продукт & $5 * 0,15=0,75$ балла & 3. Новизна продукта и окружения \\
\hline Рынок & $\begin{array}{l}5 *(0,25+0,1+0,1)=2,25 \\
\text { балла }\end{array}$ & $\begin{array}{l}\text { 2. Размер рынка } \\
\text { 5. Маркетинг, каналы продаж и партнерство } \\
\text { 4. Конкурентное окружение }\end{array}$ \\
\hline $\begin{array}{l}\text { Финансиро- } \\
\text { вание }\end{array}$ & $\begin{array}{l}5 * 0,05=0,25 \text { балла } \\
\text { Риски Потребность в дополнительных инве- } \\
\text { стициях }\end{array}$ \\
\hline
\end{tabular}

После расчета Аі показателей, выполним пятое условие квалиметрического анализа рассчитаем вес каждого базисного показателя стартапа (Pi) [1, с. 16], по формуле 1, данный расчет представлен в таблице 3.

Таблица 3.

Расчет Рі показателя

\begin{tabular}{|l|l|l|}
\hline $\begin{array}{c}\text { Базовый показатель } \\
\text { стартапа }\end{array}$ & \multicolumn{1}{|c|}{$\begin{array}{c}\text { Важность (Аі), по } \\
\text { 5-бальной шкале }\end{array}$} & $\begin{array}{c}\text { Коэффициент весомости } \\
\text { (Рі) каждого базисного } \\
\text { показателя стартапа }\end{array}$ \\
\hline Команда & $5^{*} 0,3=1,5$ балла & $30 \%$ \\
\hline Продукт & $5^{*} 0,15=0,75$ балла & $15 \%$ \\
\hline Рынок & $5^{*}(0,25+0,1+0,1)=2,25$ балла & $45 \%$ \\
\hline Финансирование & $5^{*} 0,05=0,25$ балла & $5 \%$ \\
\hline Риски & $5^{*} 0,05=0,25$ балла & \\
\hline
\end{tabular}

В результате расчета весомости каждого базисного показателя стартапа, выделенного в данной работе, можно прийти к выводу, что самым значимым показателей является - рынок, это может связано с сложностью выхода новой продукций стартапа на рынок и непонятностью для потенциального покупателя.

\section{Заключение}

В результате данной статьи была разработана квалиметрическая модель, которая применима для рейтинговая стартапа. Для этого были проведена работа такая, как: 
1. Были выделены показатели характеризующие эффективность реализации каждого из стартапов находящихся в рейтинге, такие как: команда, продукт, рынок, финансирование и риски;

2. Было построено дерево свойств по каждому базовому показателю, где наглядно можно увидеть основные критерии оценивания стартапов;

3. После был выбран экспертный метод, для анализа весомости каждого базового показателя при помощи расчета по формуле (1);

4. Далее были назначены интервалы изменения значений Рі при помощи экспертного мнения автора метода скоринга - Билла Пейна;

5. В заключение был рассчитан вес показателей каждого выделенного базисного критерия, где самым значимым из них стал критерий рынка.

Таким образом, были выявлены и оценены показатели качества стартапов и выделена структура принятия решений по их рейтингованию, при помощи руководства сравнения полученных показателей по каждому проекту в методологии квалиметрического анализа.

\section{Список литературы}

1. Белов В.М. Метод бальной оценки показателей коэффициентов весомости // Вестник федерального государственного образовательного учреждения высшего профессионального образования «Московский государственный агроинженерный университет имени В.П. Горячкина». 2010. №4 (35). С. 15-19.

2. Ключевые проблемы и передовые разработки в современной науке/ С.В. Богоченко, Р.И. Харитонов, Е.Р. Калабухов, Д.Ю. Рудаков// Сравнительный анализ современных моделей финансирования стартапа. Смоленск, 31 октября 2017. С. 171-173.

3. Войнова Ю.А., Демин С.С. Классификация рисков стартапа // Вопросы из студенческой науки. 2017. №2. С. 7.

4. Исмендеев Д.В. Квалиметрия как наука // Материалы 58-й студенческой научно-практической конференции инженерного факультета Самарской ГСХА. 2013. С. 9-12

5. Мошников Н.Э., Жукова О.В. Альтернативные методы оценки стартапов // Экономика и предпринимательство. Москва, 2017. №10-1 (87). C. 478-481. 
6. Рамазанова А.Г., Гамзатова А.М. Эффективное управление командой в стартапах // Экономика и предпринимательство. Москва, 2017. №10-1 (87). С. 684-686.

7. Родин Е.Н., Дмитриев А.Н Создание многокритериальной модели оценки качества стартапов // Российское предпринимательство. Москва, 2014. №1-135 (132) С. 128-135.

8. Скрипка В.Л., Зеленкова М.В. Информационный аспект оценок качества // Качество и жизнь. Москва, 2016. №1 (9). С. 12-16.

9. Токарев Б.Е. Модель бизнеса стартапа инновационного продукта // Вестник университета. Москва, 2018. №9. С. 55-58.

10. Фомин Ф.В. Инвестиционная оценка и анализ инвестиционной привлекательности стартапа. Методы и подходы к оценке // Инновационная экономика и менеджмент: Методы и технологи. Москва, 2017. С. 159-162.

11. Определение коэффициентов весомости. URL: https://intellect. icu/opredelenie-koeffitsientov-vesomosti-7684 (дата обращения: 22.11.2020).

12. Как оценить идею стартапа. URL: https://secretmag.ru/practice/kakocenit-ideyu-startapa-chek-list-ot-krutogo-investora.htm (дата обращения: 22.11.2020).

13. Tangkesalu A.A., Suseno J.E.Information System of Performance Assesment on Startup Business using Simple Multi-Attribute Rating Technique Exploiting Ranks (SMARTER) // E3s Web of Conferences 73, 13002. 2018, pp 2-5.

14. Kim H.; Park S.Y.; Joh W.I. Study on Technology Development Performance and Technology Commercialization Performance According to the Technology Development Capability of SMEs // Focusing on a Comparative Analysis of Technology Business Groups. 2019. pp. 63- 65.

15. Chen K., Chen S. Process Quality Analysis of Products // Int J Adv Manuf Technol. 2002. pp. 623-628.

16. Sukiasyan E. What is qualimetry? May we measure quality in points? // Scientific and Technical Libraries. 2018. no (2). pp. 113-116.

17. Litau E. Scoring method as applied to innovation project evaluation for startup support // Entrepreneurship and Sustainability. 2020. pp. 2978-2990. 
18. Laitinen, Erkki K. (2017) Profitability Ratios in the Early Stages of a Startup The Journal of Entrepreneurial Finance: Vol. 2017. no 2. pp. 1-28. 19. Qualitative approach to rating startups. URL: https://sudonull.com/ post/126128-Qualitative-approach-to-rating-startups (дата обращения: 22.11.2020).

\section{References}

1. Belov V.M. Metod bal'noy otsenki pokazateley koeffitsientov vesomosti [The method of scoring the performance of the weight coefficients]. Vestnikfederal'nogo gosudarstvennogo obrazovatel'nogo uchrezhdeniya vysshego professional'nogo obrazovaniya - Moskovskiy gosudarstvennyy agroinzhenernyy universitet imeni V.P. Goryachkina [Bulletin of the Federal state educational institution of higher professional education Moscow state Agroengineering University named after V. p. Goryachkin»]. 2010, no 4 (35), pp. 15-19.

2. Bogochenko S.V., Kharitonov R.I., Kalabukhov E.R., Rudakov D.Yu. Sravnitelnyy analiz sovremennykh modeley finansirovaniya startapa [Comparative analysis of modern startup financing models]. Klyuchevye problemy i peredovye razrabotki $v$ sovremennoy nauke [Key problems and advanced developments in modern science]. Smolensk, October 31, 2017, pp. 171-173.

3. Voynova Yu.A., Demin S.S. Klassifikatsiya riskov startapa [Classification of startup risks]. Voprosy iz studencheskoy nauki [Questions from student science]. 2017, no 2, 7 p.

4. Ismendeev D.V. Kvalimetriya kak nauka [Qualimetry as a science].Materialy 58-y studencheskoy nauchno-prakticheskoy konferentsii inzhenernogo fakul'teta Samarskoy GSKhA [Materials of the 58th student scientific and practical conference of the engineering faculty of the Samara State Agricultural Academy]. 2013, pp. 9-12

5. Moshnikov N.E., Zhukova O.V. Aleternativnye metody otsenki startapov [Innovative methods for evaluating startups]. Ekonomika i predprinimatel'stvo. [Economics and entrepreneurship]. Moscow, 2017, no. 10-1 (87), pp. 478-481.

6. Ramazanova A.G., Gamzatova A.M. Effektivnoe upravlenie komandoy $\mathrm{v}$ startapakh [Effective team management in startups]. Ekonomika i predprinimatel'stvo [Economics and entrepreneurship]. Moscow, 2017, no. 10-1 (87), pp. 684-686. 
7. Rodin E.N., Dmitriev A.N Sozdanie mnogokriterial'noy modeli otsenki kachestva startapov [Creation of a multi-criteria model for assessing the quality of startups]. Rossiyskoe predprinimatel'stvo [Russian entrepreneurship]. Moscow, 2014, no. 1-135 (132), pp. 128-135.

8. Skripka V.L., Zelenkova M.V. Informatsionnyy aspekt otsenok kachestva [Information aspect of quality assessments]. Kachestva i zhizn'[Quality and life]. Moscow, 2016, no. 1 (9), pp. 12-16.

9. Tokarev B.E. Model' biznesa startapa innovatsionnogo produkta [Innovative product startup business model]. Vestnik universiteta [University Bulletin]. Moscow, 2018, no. 9, pp. 55-58.

10. Fomin F.V. Investitsionnaya otsenka i analiz investitsionnoy privlekatel'nosti startapa. Metody i podkhody k otsenke [Investment assessment and analysis of startup investment attractiveness. Methods and approaches to evaluation]. Innovatsionnaya ekonomika i menedzhment: Metody $i$ tekhnologi [Innovative economics and management: Methods and technologists]. Moscow, 2017, pp. 159-162.

11. Opredelenie koeffitsientov vesomosti [Determination of weighting factors]. URL: https://intellect.icu/opredelenie-koeffitsientov-vesomosti-7684 (accessed 22.11.2020).

12. Kak otsenit'ideyu startapa [How to evaluate the idea of a startup]. URL: https://secretmag.ru/practice/kak-ocenit-ideyu-startapa-chek-list-ot-krutogo-investora.htm (accessed 22.11.2020).

13. Tangkesalu A.A., Suseno J.E. Information System of Performance Assesment on Startup Business using Simple Multi-Attribute Rating Technique Exploiting Ranks (SMARTER). E3s Web of Conferences 73, 13002. 2018, pp 2-5.

14. Kim H.; Park S.Y.; Joh W.I. Study on Technology Development Performance and Technology Commercialization Performance According to the Technology Development Capability of SMEs. Focusing on a Comparative Analysis of Technology Business Groups. 2019. pp. 63- 65.

15. Chen K., Chen S. Process Quality Analysis of Products. Int J Adv Manuf Technol. 2002. pp. 623-628.

16. Sukiasyan E. What is qualimetry? May we measure quality in points? Scientific and Technical Libraries. 2018. no (2). pp.113-116. 
17. Litau E. Scoring method as applied to innovation project evaluation for startup support. Entrepreneurship and Sustainability. 2020. pp. 2978-2990.

18. Laitinen, Erkki K. (2017) Profitability Ratios in the Early Stages of a Startup. The Journal of Entrepreneurial Finance: Vol. 2017. no. 2. pp. 1-28.

19. Qualitative approach to rating startups. URL: https://sudonull. com/post/126128-Qualitative-approach-to-rating-startups (accessed 22.11.2020).

\section{ДАННЫЕ ОБ АВТОРАХ}

Олейник Арина Геннадиевна, студентка второго курса магистратуры Кубанский государственный университет

ул. Ставропольская, 149, г. Краснодар, Краснодарский край, 350040, Российская Федераџия oleirina666@mail.ru

Ахмедова Милена Расуловна, кандидат экономических наук, доцент кафедры мировой экономики и менеджмента Кубанский государственный университет ул. Ставропольская, 149, г. Краснодар, 350040, Российская Федеращия mili-g1@mail.ru

\section{DATA ABOUT THE AUTHORS}

Oleynik Arina Gennadievna, second-year master student

Kuban State University

149, Stavropolskaya Str., Krasnodar, 350040, Russian Federation oleirina666@mail.ru

Akhmedova Milena Rasulovna, Candidate of Economic Sciences, Associate Professor of the Department of World economy and Management

\section{Kuban State University}

149, Stavropolskaya Str., Krasnodar, 350040, Russian Federation mili-g1@mail.ru 